\title{
Veterinary Quarterly
}

\section{Pathological characterization of primary splenic myxoid liposarcomas in three dogs}

\author{
A. Forlani, P. Roccabianca, C. Palmieri, G. Sarli, D. Stefanello, S. Santagostino, \\ C. Randi \& G. Avallone
}

To cite this article: A. Forlani, P. Roccabianca, C. Palmieri, G. Sarli, D. Stefanello, S. Santagostino, C. Randi \& G. Avallone (2015) Pathological characterization of primary splenic myxoid liposarcomas in three dogs, Veterinary Quarterly, 35:3, 181-184, DOI: 10.1080/01652176.2015.1049384

To link to this article: http://dx.doi.org/10.1080/01652176.2015.1049384

Accepted author version posted online: 08

May 2015.

Published online: 04 Jun 2015.

Submit your article to this journal $\pi$

Llll Article views: 88

View related articles $\nearrow$

View Crossmark data $\asymp$ 


\title{
CASE SERIES
}

\section{Pathological characterization of primary splenic myxoid liposarcomas in three dogs}

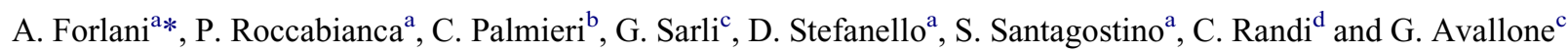 \\ ${ }^{a}$ Dipartimento di Scienze Veterinarie e Sanità Pubblica, School of Veterinary Medicine, University of Milano, Via Celoria 1020133 \\ Milano, Italy; ${ }^{b}$ School of Veterinary Science, The University of Queensland, Gatton Campus, Queensland, Australia; ${ }^{c}$ Dipartimento di \\ Scienze Mediche Veterinarie, School of Veterinary Medicine, University of Bologna, Ozzano dell'Emilia, Italy; ' Ambulatorio \\ Veterinario San Rocco, Cardano al Campo, Italy
}

(Received 19 December 2014; accepted 5 May 2015)

\begin{abstract}
Non-angiomatous-non-lymphomatous sarcomas (NANLs) represent $23 \%-34 \%$ of canine primary splenic sarcomas. Splenic liposarcomas account for $2 \%-6 \%$ of NANLs but myxoid variants are rarely reported and information on their behaviour is fragmentary. An 8-year-old male crossbreed (case 1), a 12-year-old female French bulldog (case 2), and an 11-year-old crossbreed (case 3) underwent splenectomy after the detection of a splenic nodule. Histology, histochemistry, immunohistochemistry, and transmission electron microscopy (TEM) were performed. Bundles of spindle-to-polygonal cells containing occasional cytoplasmic oil-red-O positive vacuoles embedded in an Alcian blue-positive extracellular matrix were observed. Aggregates of round cells were detected in cases 1 and 3. All tumours were vimentin positive and actin, desmin, Factor VIII, and S100 negative. The TEM evidenced different maturational stages of adipose cells (lipoblasts, intermediate, and undifferentiated). All the cases developed hepatic metastases and were euthanized. Disease free interval was 2 months in cases 1 and 3, and 21 months in case 2 . The presence of a neoplastic embolus in case 1 and areas of round cell differentiation in cases 1 and 3 represented the sole prognostic indices.
\end{abstract}

Keywords: dog; electron microscopy; histochemistry; immunohistochemistry; myxoid liposarcoma; spleen

Angiosarcoma and lymphoma are the most common primary canine splenic tumours, while the so-called splenic non angiomatous-non lymphomatous sarcomas (NANLs) are uncommon neoplasms with uncertain characterization (Weinstein et al. 1989; Spangler \& Culbertson 1992; Spangler et al. 1994; Day et al. 1995). The canine NANLs account for $23 \%$ to $34 \%$ of primary splenic neoplasms, and most frequently include fibrosarcoma, leiomyosarcoma, and undifferentiated sarcoma, while liposarcomas (LPS) are rare (Weinstein et al. 1989; Spangler \& Culbertson 1992; Spangler et al. 1994; Day et al. 1995). LPS are infrequent soft tissue neoplasms with a reported incidence lower than $0.5 \%$ of cutaneous and subcutaneous canine and feline tumours (Goldschmidt \& Shofer 1992). Primary splenic LPS have been included in large caseloads of mesenchymal tumours of the canine spleen accounting for $2 \%-6 \%$ of NANLs and, consequently, specific information regarding LPS histotypes and their biology are fragmentary (Weinstein et al. 1989; Spangler \& Culbertson 1992; Spangler et al. 1994; Day et al. 1995). In man, LPS are characterized by four histological subtypes: atypical lipomatous tumour/well-differentiated, myxoid, dedifferentiated, and pleomorphic, each bearing a distinctive prognosis (Chang et al. 1989; Goldblum et al. 2013). Well-differentiated LPS, the most common variant of LPS in man, are locally aggressive but rarely metastasize, while the less frequent dedifferentiated LPS have a high recurrence rate and metastasize frequently (Goldblum et al. 2013). The second most common type is the myxoid variant, that develops metastases in up to $58 \%$ of cases (Goldblum et al. 2013).

In dogs, three histotypes of soft tissue LPS have been described: well differentiated, myxoid, and pleomorphic (Hendrick et al. 1998). Pleomorphic LPS seems to be the most aggressive variant, although a distinctive behavioural pattern has not been conclusively demonstrated (Baez et al. 2004; Gross et al. 2005). The aim of this report is to describe the pathological and clinical features of three cases of canine primary splenic myxoid LPS.

Three dogs, two male crossbreed, 8- and 11-years-old (case 1 and 3), respectively, and a 12-year-old female spayed French bulldog (case 2), underwent abdominal ultrasonography after chronic vomiting (cases 1 and 3) and for a check after several months of the prednisone therapy administered to control a severe atopic dermatitis (case 2). Ultrasonography revealed a single splenic mass and the dogs underwent splenectomy. Splenic masses were $15 \times 15 \mathrm{~cm}$ in diameter (case 1), $6.5 \times 7 \mathrm{~cm}$ (case 2), $15 \times 10 \mathrm{~cm}$ (case 3 ), white to light tan, glistering, greasy, and mucoid on the cut surface.

Tissue samples from the three masses were formalinfixed, paraffin-embedded and routinely processed for histology. Alcian ( $\mathrm{pH}$ 3.1)-Periodic Acid Schiff (PAS) stain was performed on selected sections. Formalin-fixed specimens were also snap-frozen in liquid nitrogen and cryostat sections were stained with oil red-O. Immunohistochemistry was performed in all cases on poly-lysine coated slides using the avidin-biotin-peroxidase method and specific

*Corresponding author. Email: giancarlo.avallone@gmail.com; giancarlo.avallone@unibo.it 
primary mouse monoclonal antibodies against vimentin (Dako, clone 3B4, dilution 1:2000), smooth muscle actin (Dako, clone 1A4, dilution 1:2000), desmin (Novocastra, clone DE-R-II, dilution 1:200), and rabbit polyclonal antibodies anti-factor VIII-related antigen (Dako, Cat. $\mathrm{N}^{\circ}$ A0082, dilution 1:200), and S100 (Dako, Cat. $\mathrm{N}^{\circ}$ Z0311, dilution 1:10000). Heat-induced antigen retrieval (microwave, 10 minutes, $\mathrm{pH} 6$ citrate buffer) was applied for immunohistochemistry for vimentin, smooth muscle actin, desmin, and factor VIII-related antigen. No antigen retrieval was applied for S100. For transmission electron microscopy (TEM), tissue fragments from cases nos. 1 and 2 were fixed in $2.5 \%$ glutaraldehyde, post-fixed in $1 \%$ $\mathrm{OsO}_{4}$, and embedded in epoxy resin. Thin sections $(70$ $\mathrm{nm})$ from selected areas of interest were stained with lead citrate and uranyl acetate. No additional samples from case 3 were available for the ultrastructural analysis.

Microscopically, all tumours were composed of haphazardly arranged, spindle-to-stellate cells embedded in a pale eosinophilic matrix. Cells were $15-25 \mu \mathrm{m}$ in diameter, with a high nucleus/cytoplasmic ratio $(\mathrm{N} / \mathrm{C})$, variable amount of pale cytoplasm rarely containing sharply demarcated, clear cytoplasmic vacuoles. Nuclei were round to oval, with granular to vesicular chromatin and an occasionally visible central, round, nucleolus. In case 1 , the neoplasm was infiltrative, non-encapsulated and multifocally characterized by large lacunae lined by neoplastic cells and filled with lightly eosinophilic material (Figure 1(a)). Rare multinucleated neoplastic cells and multifocal areas of necrosis were present. In more than $20 \%$ of the tumour (semiquantitative evaluation of all the haematoxylin and eosin-stained sections available at intermediate magnification), cells were round shaped, with higher N/C ratio and small amount of cytoplasm. One mitotic figure was identified in 10 high power fields (HPF). After serial sectioning, a single neoplastic intravascular embolus was detected. In case 2, the tumour was well demarcated, partially encapsulated, expansile, multilobular, and confined within the splenic parenchyma. A plexiform network of thin-walled capillaries branching at 45 to 90 degree angles was the typical histological findings. Occasional lacunae similar to case 1 were observed. Differentiated lipocytes were observed in $20 \%$ of the mass. Necrosis and haemorrhages were absent and mitoses were 16 in $10 \mathrm{HPF}$. In case 3 , the tumour was non-encapsulated, focally infiltrative, and characterized by large lacunae similar to case 1 . Round cell areas occupied more than $20 \%$ of the examined neoplasm. Large areas of necrosis were detected and 18 mitotic figures were observed in $10 \mathrm{HPF}$. The extracellular matrix and the material within the lacunae were Alcian blue-positive and PAS-negative, consistent with acidic mucins (Figure 1(b)). Lipocytes, lipoblasts, and scattered

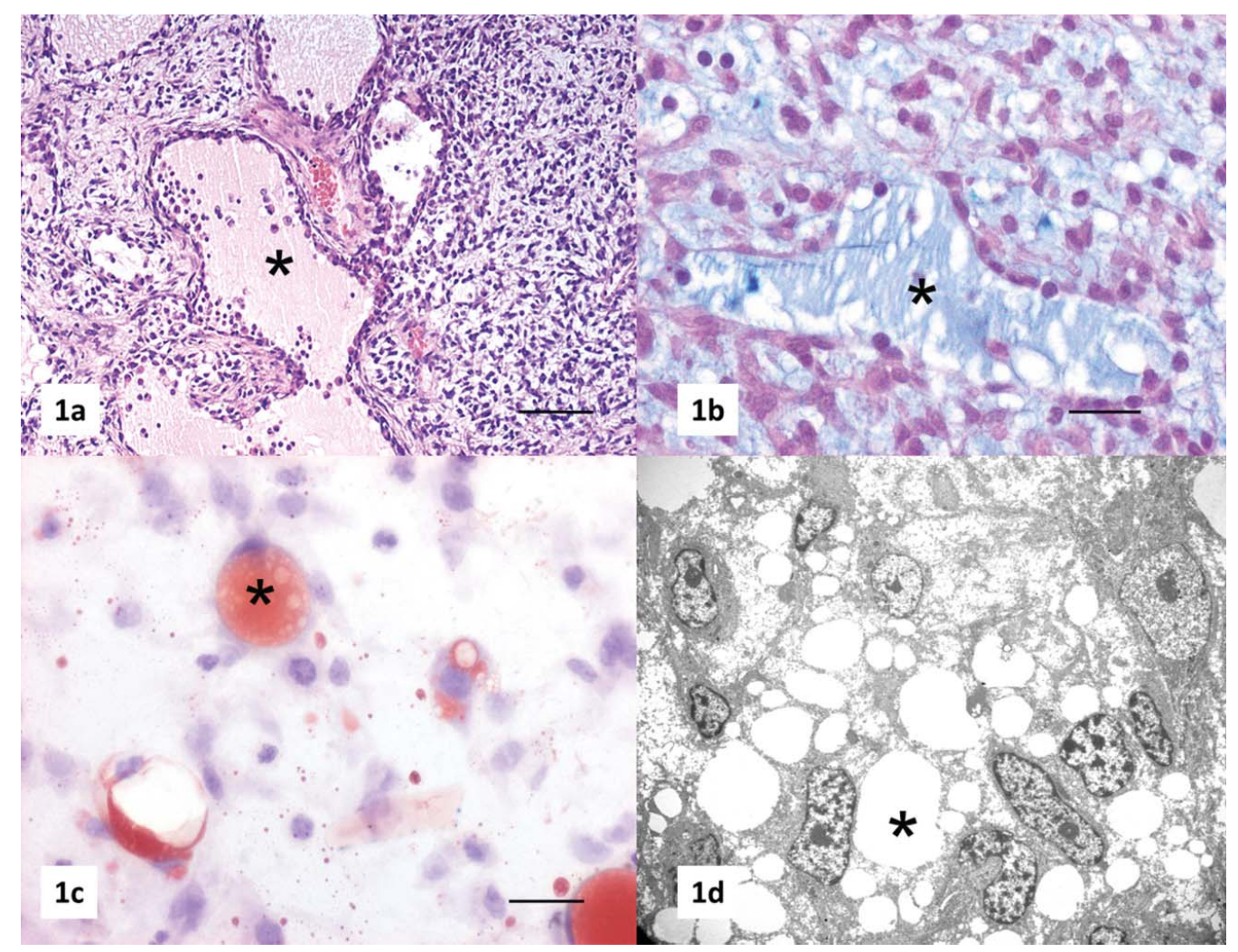

Figure 1. (a) Splenic mass from an 8-year-old male crossbreed dog (case 1). The neoplasm is composed by stellate to round cells lining large lacunae filled with lightly eosinophilic material (asterisk). Haematoxylin and eosin. Bar $=120 \mu \mathrm{m}$. (b) Splenic mass from an 8year-old male crossbreed dog (case 1). The neoplasm is composed by stellate cells with optically empty cytoplasmic vacuoles. Note the lacunae lined by neoplastic cells and the Alcian blue-stained extracellular matrix (asterisk). Alcian blue. Bar $=50 \mu \mathrm{m}$. (c) Splenic mass from a 12-year-old female French bulldog (case 2). The intracytoplasmic vacuoles within neoplastic cells are Oil-red-O positive (orange colour) (asterisk), confirming the lipid content of neoplastic cells. Oil-red-O. Bar $=50 \mu \mathrm{m}$. (d) Aggregate of lipoblastic cells containing high number of electronlucent intracytoplasmic lipid droplets (asterisk). TEM, uranyl acetate and lead citrate. Original magnification = 3000x. 
neoplastic spindle cells contained oil red-O positive cytoplasmic vacuoles (Figure 1(c)). Neoplastic cells were vimentin positive and smooth muscle actin, desmin, Factor VIII-related antigen and S100 negative. According to the histology and soft tissue sarcomas, grading all tumours were diagnosed as grade II myxoid LPS

TEM performed on cases 1 and 2 identified three types of neoplastic cells: primitive undifferentiated mesenchymal cells, intermediate cells, and fully developed lipoblasts. Intermediate cells and lipoblasts were respectively the most and the less represented cell types. Neoplastic cells were embedded in an amorphous matrix occasionally coating and clinging on the cells. Undifferentiated cells were round to polygonal to spindle, had few intracytoplasmic organelles, no lipid vacuoles and a poorly developed basal lamina or basal lamina remnants. Intermediate cells were spindle to stellate with variable numbers of mitochondria and rough endoplasmic reticulum (RER) and without sufficient lipid vacuoles to be considered lipoblasts. In the early stages of development, they were frequently seen in apposition to capillaries. A basal lamina encircled intermediate cells, especially those nearest to the capillary walls. Lipoblasts were round to polygonal, had numerous mitochondria, scant RER, and a poorly formed basal lamina or scarce basal lamina remnants. Their cytoplasm contained variably sized lipid vacuoles with different electrondensity (Figure 1(d)). The combination of all these results confirmed the diagnosis of grade II myxoid LPS in both cases.

All the cases developed hepatic metastases detected by ultrasonography and were euthanized because of poor prognosis. Disease-free interval was 2 month in cases 1 and 3, and 21 months in case 2. Case 1 underwent necropsy that confirmed multiple metastases in the liver and in the sternal lymph node.

To the best of our knowledge, this study represents the most comprehensive pathological characterization of canine splenic myxoid LPS. Moreover, no data on the biological behaviour of these neoplastic conditions have been previously reported in dogs. Canine LPS arises most frequently in the subcutis, and histological grade is considered prognostic (Dennis et al. 2011). However, grade was not predictive of the aggressive behaviour in case 1. A study by Baez et al. (2004) on 56 cases of canine LPS highlighted that dogs undergoing wide surgical excision had significantly longer survival times than dogs undergoing marginal excision (Baez et al. 2004). However, splenectomy did not correlate with the clinical outcome in the current cases. The most important prognostic factor for NANLs is mitotic index, and more than 9 mitoses in 10 HPF have been correlated with shorter survival times (Spangler et al. 1994). However, in case 1, the mitotic index was lower than the cutoff set by the grading system for NANLs, and this parameter did not envisage the aggressive behaviour. Conversely, case 2 was characterized by a longer survival time despite a mitotic index higher than 9. The correlation between the mitotic index and prognosis has not been well defined for visceral LPS and, as observed in our cases, the number of mitoses in splenic LPS may not be prognostically significant compared to other NANLs. This represents a novel finding since few splenic LPS ( $2 \%-6 \%$ of NANL) have been included in previous caseloads and in none of them the relevance of the histological grade can be clearly inferred (Weinstein et al. 1989; Spangler \& Culbertson 1992; Spangler et al. 1994; Day et al. 1995). Additionally, it should be emphasized that the two cases with shorter survival time were characterized by a large round cell component (case 1 and 3). This histological feature is considered highly predictive of metastasis and poor prognosis in myxoid LPS in man (Goldblum et al. 2013). A prognostic study on a larger number of cases should be performed in order to confirm these observations. Further parameters indicative of an aggressive behaviour in cases 1 and 3 were the larger size, the infiltrative growth, the randomly scattered areas of necrosis, and the presence of a neoplastic embolus (case 1).

In case 2 , the clear demarcation from the normal parenchyma, the partial encapsulation, and the presence of well-differentiated lipocytes were distinctive and could be considered indicative of a more differentiated and less aggressive tumour. In all cases, the application of ancillary diagnostic techniques was pivotal to confirm the diagnosis. The diagnostic hallmark of human myxoid LPS in soft tissues is the detection of a specific FUS-DDIT3 fusion oncogene, resulting from a $\mathrm{t}(12,16)(\mathrm{q} 13, \mathrm{p} 11)$ chromosome translocation (Goldblum et al. 2013). However, no data are available regarding the cytogenetic anomalies in canine LPS. Therefore, the diagnosis was exclusively and conclusively based on the histological, immunohistochemical, histochemical, and ultrastructural features. Immunohistochemistry ruled out the two most common types of splenic sarcoma (haemangiosarcoma and leiomyosarcoma), while histochemistry and TEM identified the lipogenic origin of neoplastic cells with the myxoid component. Additionally, TEM identified characteristic intermediate cells in apposition to vessels, so paralleling the differentiation gradient described in the human myxoid LPS that suggests the derivation of these types of tumour from undifferentiated perivascular cells, as occurs during the normal development of the white adipose tissue (Battiflora and Nunez-Alonso 1980). The gross morphology, multiple sampling, and sectioning for histological, histochemical, and immunohistochemical stains as well as TEM should be combined to achieve the correct diagnosis and subtype in canine undifferentiated splenic sarcomas. The prognostic significance of mitotic index, grade, and subtype should be evaluated on a larger number of visceral LPS and sarcomas to provide insights on and identify the most accurate factors for their correct grading.

\section{Acknowledgements}

We thank Prof Malcolm Jones and Dr Erica Lovas (School of Veterinary Science, The University of Queensland, Gatton campus, Gatton, Queensland, Australia) for the electron microscopy assistance.

\section{Disclosure statement}

No potential conflict of interest was reported by the authors. 


\section{References}

Baez JL, Hendrick MJ, Shofer FS, Goldkamp C, Sorenmo KU. 2004. Liposarcomas in dogs: 56 cases (1989-2000). J Am Vet Med Assoc. 224:887-891.

Battifora H, Nunez-Alonso C. 1980. Myxoid liposarcoma: study of ten cases. Ultrastruct Pathol. 1:157-169.

Chang HR, Hajdu SI, Collin C, Brennan MF. 1989. The prognostic value of histologic subtypes in primary extremity liposarcoma. Cancer. 64:1514-1520.

Day MJ, Lucke VM, Pearson H. 1995. A review of pathological diagnoses made from 87 canine splenic biopsies. J Small Anim Pract. 36:426-433.

Dennis MM, McSporran KD, Bacon NJ, Schulman FY, Foster RA, Powers BE. 2011. Prognostic factors for cutaneous and subcutaneous soft tissue sarcomas in dogs. Vet Pathol. 48:73-84.

Goldblum JR, Folpe AL, Weiss SW. 2013. Enzinger \& weiss's soft tissue tumors. 6th ed. Philadelphia (PA): Elsevier Saunders. Chapter 15, Liposarcoma; p. 484-523.

Goldschmidt MH, Shofer FS. 1992. Skin tumors in the dog and cat. Oxford: Pergamon Press. Chapter 24, Cutaneous lipoma and liposarcoma; p. 192-203.
Gross TL, Ihrke PJ, Walder EJ, Affolter VK. 2005. Skin diseases of the dog and cat: clinical and histopathologic diagnosis. 2nd ed. Oxford: Blackwell Science. Chapter 30, Lipocytic Tumors; p. 766-772.

Hendrick MJ, Mahaffey EA, Moore FM, Vos JH, Walder EJ. 1998. Histologic classification of mesenchymal tumors of skin and soft tissues of domestic animals. Washington (DC): Armed Forces Institute of Pathology. p. 19-20.

Spangler WL, Culbertson MR. 1992. Prevalence, type, and importance of splenic diseases in dogs: 1,480 cases (1985-1989). J Am Vet Med Assoc. 200:829-834.

Spangler WL, Culbertson MR, Kass PH. 1994. Primary mesenchymal (nonangiomatous/nonlymphomatous) neoplasms occurring in the canine spleen: anatomic classification, immunohistochemistry, and mitotic activity correlated with patient survival. Vet Pathol. 31:37-47.

Weinstein MJ, Carpenter JL, Schunk CJ. 1989. Nonangiogenic and nonlymphomatous sarcomas of the canine spleen: 57 cases (1975-1987). J Am Vet Med Assoc. 195:784-788 